\title{
Early Onset Alcohol Use and Self-Harm: A Discordant Twin Analysis
}

\author{
Lauren R. Few, Kimberly B. Werner, Carolyn E. Sartor, Julia D. Grant, Timothy J. Trull, \\ Matthew K. Nock, Kathleen K. Bucholz, Sarah K. Deitz, Anne L. Glowinski, Nicholas G. Martin, \\ Elliot C. Nelson, Dixie J. Statham, Pamela A. F. Madden, Andrew C. Heath, \\ Michael T. Lynskey, and Arpana Agrawal
}

\begin{abstract}
Background: Self-harm has considerable societal and economic costs and has been extensively studied in relation to alcohol involvement. Although early onset alcohol use (EAU) has been causally linked to maladaptive clinical outcomes, its association with self-harm is less well characterized. This study aimed to further examine the link between EAU and both nonsuicidal self-injury (NSSI) and suicide attempt (SA), and elucidate shared familial and causal/individual-specific pathways that explain this co-occurrence.

Methods: Using data from 6,082 Australian same-sex twin pairs (1,732 monozygotic [MZ] and 1,309 dizygotic [DZ]), ages 23 to 40, we examined prevalence rates of NSSI and SA among twin pairs concordant and discordant for EAU. Conditional logistic regression, controlling for early clinical covariates and the influence of zygosity on EAU, was used to examine the odds ratio (OR) of self-harm within twin pairs discordant for EAU.

Results: Prevalence rates of both NSSI and SA were highest among twin pairs concordant for EAU and for twins who reported EAU within discordant twin pairs. Results from discordant twin analyses revealed nearly 4-fold increased odds of SA for the twin who endorsed EAU, and this OR was equal across MZ and DZ twins. EAU also was associated with elevated odds of NSSI (OR = 7.62), although this was only the case for DZ twins in discordant pairs.

Conclusions: The equivalent increase in odds of SA for both MZ and DZ twins suggests that causal or individual-specific influences explain the link between EAU and SA. For NSSI, elevated odds for DZ twins and nonsignificant findings for $\mathrm{MZ}$ twins implicate correlated genetic factors in the association between EAU and NSSI. Future studies should test mechanisms through which EAU may causally influence SA, as well as examine whether genetic risk for third variables (e.g., negative urgency, stress reactivity) may explain the genetic overlap between EAU and NSSI.
\end{abstract}

Key Words: Early Alcohol Use, Self-Harm.

$\mathrm{S}$ ELF-harm can be broadly categorized into 2 main forms: suicide attempt (SA) and nonsuicidal self-injury (NSSI), the latter of which is defined as deliberate destruction or alteration of body tissue in the absence of suicidal intent

From the Department of Psychiatry (LRF, CES, JDG, KKB, SKD, $A L G, E C N, P A F M, A C H, A A)$, Washington University School of Medicine, Saint Louis, Missouri; George Warren Brown School of Social Work $(K B W)$, Washington University, Saint Louis, Missouri; Department of Psychiatry (CES), Yale University School of Medicine, West Haven, Connecticut; Department of Psychological Sciences (TJT), University of Missouri, Columbia, Missouri; Department of Psychology (MKN), Harvard University, Cambridge, Massachusetts; QIMR Medical Research Institute (NGM), Brisbane, Queensland, Australia; School of Social Sciences (DJS), University of the Sunshine Coast, Sippy Downs, Queensland, Australia; and Institute of Psychiatry, Psychology \& Neuroscience (MTL), King's College London, London, United Kingdom.

Received for publication May 19, 2015; accepted August 26, 2015.

Reprint requests: Lauren R. Few, PhD, Department of Psychiatry, Washington University School of Medicine, 660 S. Euclid Ave, Campus Box 8134, St. Louis, MO 63110; Tel.: 314-286-2205; Fax: 314-286-2213; E-mail:fewl@psychiatry.wustl.edu

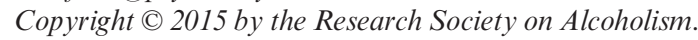

DOI: 10.1111/acer.12889
(Nock and Favazza, 2009). Self-harm is the 18th leading contributor to the global disease burden and 13th highest contributor to years of life lost globally (GBD 2010 Country Collaboration, 2013). Strikingly, SA is the second most common cause of death among individuals ages 10 to 24 (Patton et al., 2009). The cross-national lifetime prevalence of SA among adults is estimated at $3.1 \%$ (Nock et al., 2008), and meta-analysis has estimated that $5.5 \%$ of adults have engaged in lifetime NSSI, although due to the considerable heterogeneity in the methodology used to study NSSI (Swannell et al., 2014), estimates vary from study to study. In adolescent samples, prevalence rates are higher with $4.1 \%$ reporting SA (Nock et al., 2013) and approximately 17.2\% of adolescents endorsing NSSI (Swannell et al., 2014). Although NSSI and SA are distinct phenotypically, they frequently co-occur, which is evident in both clinical and community samples (Bebbington et al., 2010; e.g., Nock et al., 2006). Furthermore, NSSI has been shown to be a risk factor for SA (for a review, see Hamza et al., 2012). More importantly, they both share similar clinical correlates, which further supports the notion that there may be common mechanisms underlying their co-occurrence. 
Alcohol involvement has been strongly implicated in the epidemiology and clinical course of self-harm. Several crosssectional studies have demonstrated a significant relationship between both adolescent and adult alcohol involvement (e.g., frequency of use; alcohol use disorder) and self-harm (Bagge and Sher, 2008; Conner et al., 2014; Glowinski et al., 2001; Moller et al., 2013). Another prominent area of research has focused on acute use of alcohol prior to suicide, finding substantially increased risk for intoxication prior to suicide completion relative to nonsuicidal controls (Kaplan et al., 2014). A few prospective studies have further demonstrated that distal adolescent alcohol use predicts both SA (Stewart et al., 2001) and NSSI (Tuisku et al., 2014). These findings are consistent with adult studies demonstrating that alcohol abuse and dependence predict prospective repetition of self-harm (Larkin et al., 2014).

Despite elevated rates of self-harm in adolescence, a particular aspect of alcohol involvement that has received somewhat less attention in relation to self-harm is early onset alcohol use (EAU). In general, EAU has been associated with a host of maladaptive outcomes, including alcohol problems and sexual risk-taking (Stueve and O'Donnell, 2005), as well as lower education attainment (Grant et al., 2012). It has also been linked more specifically to increased risk for SA (Bossarte and Swahn, 2011; Swahn and Bossarte, 2007; Swahn et al., 2010) and comorbid reports of SA and physical fighting among high school students (Swahn et al., 2013). Far fewer studies have examined EAU and NSSI. Among them is Giletta and colleagues' multinational study (2012), which found no association between frequency of binge drinking and NSSI among adolescents in 3 countries. However, this study did not differentiate between early versus late adolescent initiators of alcohol use.

There are several potential theoretical explanations for the association between EAU and self-harm. One possibility is that these 2 phenotypes reflect shared genetic liability. There is inconsistent evidence for genetic influences on EAU (Agrawal et al., 2009; Prescott and Kendler, 1999; RichmondRakerd et al., 2014; Sartor et al., 2009a) and evidence that genetic factors influence NSSI (Maciejewski et al., 2014) as well as SA (Glowinski et al., 2001). It is also possible that the overlap between EAU and self-harm reflects an underlying personality disposition, such as negative urgency (Dir et al., 2013) or that these behaviors may be a consequence of other known risk factors, such as childhood sexual abuse (Moller et al., 2013; Sartor et al., 2013). An alternative hypothesis that has not been explored is that the link between EAU and self-harm is causal, such that initiating drinking at a young age independently and directly increases the likelihood of subsequent self-harm. A median onset of SA in the mid-20s has been retrospectively reported in adult samples (Kessler et al., 1999), which is typically after initiation of alcohol use. In adolescent samples, the median age of onset of SA (Nock et al., 2013) and NSSI (Klonsky, 2011) is 14, which is within the same age range as has been reported for EAU (Hingson and White, 2014; Sartor et al., 2009b), suggesting that a causal relationship is plausible. The existing work linking these phenotypes, however, is cross-sectional, thus minimizing the ability to disentangle causality. In addition, it has focused solely on SA compared to NSSI. Last, these studies are unable to control for genetic risk factors to study causality.

Even in the absence of longitudinal data, cross-sectional twin samples offer a unique opportunity to elucidate potential mechanisms underlying the association between EAU and self-harm. Specifically, the relative role of shared genes and individual-specific environmental factors, which can exert putative causal influence, can be disentangled by selecting pairs of twins discordant for EAU. Monozygotic (MZ) twins share $100 \%$ of their genes and their shared familial environment, while dizygotic (DZ) twin pairs share $50 \%$ of their genes (and $100 \%$ of their shared familial environment). Therefore, an attenuation of the association between EAU and self-harm with increasing levels of genetic identity-by-descent (e.g., Unrelated pairs > discordant DZ pairs > discordant MZ pairs) reflects the role of shared genetic influences. Evidence for potentially causal effects can be gleaned by examining the extent to which the likelihood of self-harm is elevated in the early alcohol using twin in discordant MZ pairs. If the association is significant, then factors other than those shared by a pair of MZ twins contribute to the relationship. These factors could be causal (i.e., EAU directly causes self-harm) or related to a mediating individual-specific environmental factor. For example, EAU has been shown to result in maladaptive brain development and deficits in executive functioning (for a review, see Guerri and Pascual, 2010), which may contribute to subsequent self-harming behaviors. Thus, although the presence of longitudinal data is critical to establishing temporality and causation, the discordant twin approach can offer a window into possible nongenetic pathways for further study.

In this study, we use data from a large population-representative sample of adult Australian twins $(N=9,591$, ages 23 to 40 years) to examine whether (i) EAU is associated with SA and NSSI, and further (ii) whether this association persists in 698 discordant twins pairs (363 MZ and $335 \mathrm{DZ}$ ) after accounting for other early confounders (e.g., marijuana use, childhood sexual abuse, conduct disorder, etc.).

\section{MATERIALS AND METHODS}

\section{Participants}

The sample consisted of 9,591 adult twins from the Australian Twin Registry assessed in 2 separate cohorts. The first cohort of twins was born between 1964 and 1971 and interviewed between 1996 and 2000. The second cohort was born between 1972 and 1979 and interviewed between 2005 and 2009. More information on interview procedures and participant demographics are documented elsewhere (Lynskey et al., 2003; for more information on interview procedures and participant demographics, see Lynskey et al., 2012). In this study, we used data only from the 7,334 same-sex MZ and DZ twins. Lifetime nondrinkers $(N=125)$ and singletons $(N=1,127)$ were removed for the discordant twin analyses leaving 6,082 twin individuals $(3,464 \mathrm{MZ}$ and 2,618 DZ), ages 23 to 40 
$($ mean age $=30.47), 59 \%$ of whom were female. For the bivariate twin modeling, singletons were included in the analyses (MZF: 1,080 pairs + 287 unpaired; MZM: 652 pairs +295 unpaired; DZF: 800 pairs + 254 unpaired; DZM: 504 pairs + 292 unpaired).

\section{Measures}

All variables were assessed via interview using the Australian version of the Semi-Structured Assessment of the Genetics of Alcoholism (SSAGA-OZ).

Early Onset Alcohol Use. To assess EAU, participants who reported ever drinking in their lifetime were asked, "How old were you the first time you had a full drink of beer, wine or spirits?" Participants were previously told that a full drink is defined as "a standard can or stubbie of beer, a glass of wine, a nip of spirits, or any other kind of drink with alcohol in it." The age threshold for determining EAU was based on examination of previous research reporting poor clinical outcomes associated with initiation of alcohol use before age 15 (see Hingson and White, 2014; for a review, see Sartor et al., 2009b) and the frequency distribution for initiation of alcohol use in this study. Therefore, EAU was operationalized as drinking prior to age 15 which was associated with a prevalence of $20.42 \%$ ( $\leq 13: 12.14 \% ; \leq 15: 36.77 \% ; \leq 16: 60.28 \%$ ). Therefore, participants received a 1 if they reported their first drink prior to age 15 (i.e., 14 years old or earlier), and a 0 if their first drink was at age 15 or older.

Self-Harm. The 2 self-harm variables were also dichotomously coded as 0 ("No") or 1 ("Yes"). For SA, participants responded to the question, "Have you ever tried to take your own life?" This question was asked of all participants, not just those who reported depressed mood or suicidal thoughts. Following a positive endorsement of SA, age of onset for the first occurrence was also assessed. To maximize the ability to make causal inferences regarding the association between EAU and self-harm, participants were excluded if they reported an age of SA onset prior to the age of onset of alcohol use. This resulted in 49 individuals being excluded for SA analyses. For NSSI, participants were asked, "(Other than when you tried to take your own life), have you ever hurt yourself on purpose, for example, by cutting or burning yourself?" Age of onset was not assessed for NSSI. For participants endorsing SA or NSSI, additional questions were asked including the method used in the most serious SA (e.g., medication ingestion, hanging, etc.) as well as the individual's emotional/behavioral state prior to the SA (i.e., feeling depressed, drinking heavily, feeling extremely good or high, using drugs, having strange thoughts or experiences, or seeing visions). In a subset of individuals, methods of NSSI (e.g., cutting, burning, etc.) were also assessed.

Clinical Covariates. Several clinical covariates were included in the analyses. In order for a covariate to be considered present, the age of onset had to occur either prior to or within the same year as the age of onset of alcohol use, thereby ensuring that it also occurred in the same year or prior to the age of onset of SA (although for NSSI, this could not be determined because of the lack of age of onset assessment). Dichotomous covariates included: (i) Depressed mood was assessed by asking participants if there has "ever been 2 weeks or more when you were depressed or down most of the day, nearly every day." (ii) Anhedonia was operationalized as a 2-week period of being "a lot less interested in most things or unable to enjoy the things you need to enjoy." (iii) Childhood sexual abuse was assessed by asking participants if they were ever forced into sexual intercourse or sexual activity before the age of 18. (iv) Family history of suicide was determined by having participants report on whether one of their relatives had ever committed suicide. (v) Family history of excessive alcohol use was assessed by asking participants if they have ever felt that either their biological mother or father was an excessive drinker. Notably, family history of suicide and excessive drinking were not included in the discordant twin analyses, because twins are matched for family history. In addition, a (vi) Risky Behavior count variable was created via summation of 4 endorsed risky behaviors including marijuana use, operationalized as using the drug, even once, during the lifetime; early smoking, defined as smoking a cigarette at least 1 day a week for a period of 3 weeks or more; early sex, assessed by asking participants the age of first voluntary sexual intercourse; and conduct problems, defined as the presence of 1 or more conduct disorder symptoms (e.g., physical fighting, hurting animals, shoplifting). From this count variable, 4 dummy coded variables were created (all 4; 3 only; 2 only; 1 only). Last, gender was also included as a covariate but not in the discordant twin analyses, because twins are matched for gender.

Data Analysis. All of the data preparation and descriptive analyses were conducted using SAS/GRAPH ${ }^{\circledR}$ (9.2 Reference; SAS Institute Inc., Cary, NC). Stata Statistical Software (Release 9; StataCorp., College Station, TX) was used for logistic regression analyses examining the association between EAU and self-harm variables. First, self-harm was characterized by examining the overlap between SA and NSSI, its association with EAU (adjusting for family structure), as well as methods of SA and NSSI among those with and without a history of EAU. Correlations between self-harm and EAU and other clinical covariates were then examined to identify variables to include in subsequent analyses. The prevalence of EAU and clinical covariates was also reported in individuals who endorsed self-harm versus those individuals who denied a lifetime history of self-harm. We then examined the prevalence of self-harm across 4 groups: (i) twins concordant for EAU (i.e., prior to age 15), (ii) twins concordant for not using alcohol before the age of 15, (iii) individuals from discordant twin pairs who reported EAU, and (iv) individuals from discordant twin pairs who did not drink before age 15. Prevalence of clinical covariates across these groups was also examined, and logistic regression was used to test whether the association between EAU and self-harm was significant after controlling for these covariates. Gender was also included as a covariate in this model.

The discordant MZ and DZ twin pairs were then utilized in conditional logistic regression analyses to examine the link between EAU and self-harm while accounting for shared predispositions. Importantly, to test whether associations were significantly different in discordant DZ and MZ pairs (the latter being critical for demonstration of causal/individual-specific environmental effects), an interaction term between zygosity (MZ or not) and EAU status was entered into the adjusted models. If the interaction was significant, then the unadjusted models were re-run in MZ and DZ twins separately to examine differences in odds ratios (ORs).

\section{RESULTS}

\section{Characterizing Self-Harm and Its Associations with EAU}

The tetrachoric correlation between NSSI and SA was $0.53(p<0.01)$. Of the 6,082 twins retained in the final sample, 247 (4.1\%) reported a history of NSSI only, $144(2.4 \%)$ reported SA only, and $59(1.0 \%)$ participants endorsed a history of both NSSI and SA. The mean age of SA onset was 21.12 years $($ median $=20.0)$. EAU was significantly associated with both NSSI and SA, with ORs of 2.41 (95\% confidence interval $[\mathrm{CI}]: 1.89$ to $3.07, p<0.01)$ and $2.35(95 \%$ 
CI: 1.75 to $3.15, p<0.01$ ), respectively (see Table $\mathrm{S} 1$ for parameter estimates and test statistics).

There was no evidence for significant differences in features of SA or NSSI in those reporting EAU relative to the full sample. Across individuals reporting EAU and those with later onset, the most common methods of NSSI were cutting $(67.88 \%)$ and burning $(21.17 \%)$, while the most commonly reported methods for SA were ingestion of medication $(56.10 \%)$ and bleeding, such as cutting wrists or stabbing self $(20.70 \%)$. Furthermore, for SA, 56\% of individuals reporting EAU (vs. $52.30 \%$ of later onset users) reported intent to die and $45.33 \%$ (vs. $53.91 \%$ of later onset users) of individuals reported requiring treatment following the SA. Depressed mood, followed by being drunk, were the 2 most commonly reported emotional states prior to SA in the sample, regardless of EAU.

\section{Prevalence of EAU, Self-Harm, and Clinical Covariates}

Table 1 provides the prevalence of EAU and clinical covariates among twin individuals with and without a history of self-harm. Among those reporting NSSI, the prevalence of EAU was $36.9 \%$ but only $19.6 \%$ for individuals without a history of NSSI. The prevalence of EAU was $37.0 \%$ and $20.0 \%$ for those with and without a history of SA, respectively.

All clinical covariates were significantly associated with self-harm variables (see Table S2 for tetrachoric correlations and chi-square test results), with the exception of depressed mood and having endorsed only 1 risky behavior. Significant correlations ranged from 0.09 ( 2 risky behaviors) to 0.38 (anhedonia, regardless of depressed mood) for NSSI, and from 0.13 (2 risky behaviors) to 0.46 (childhood sexual abuse) for SA. Females reported significantly more SA relative to males, $\chi^{2}(1,6033)=7.23, p<0.01$, but there were no significant gender differences for NSSI. For all clinical covariates except the dummy coded variable encompassing individuals reporting only risky behavior, prevalence rates were higher among those with a history of self-harm (i.e., 95\% confidence limits did not overlap). Again, this was not the case for depressed mood, which was removed from subsequent analyses due to the lack of an association with the self-harm variables. ${ }^{1}$ The ORs between EAU and self-harm remained significant when adjusting for these clinical covariates (NSSI: $\mathrm{OR}=2.71,95 \% \mathrm{CI}: 2.10$ to $3.51 ; \mathrm{SA}: \mathrm{OR}=2.56,95 \% \mathrm{CI}$ : 1.89 to 3.54; see Table S1 for parameter estimates and test statistics).

The prevalence of SA and NSSI in the full sample most closely approximated estimates from individuals (in concordant and discordant twin pairs) who did not endorse EAU

\footnotetext{
${ }^{1} \mathrm{~A}$ dichotomous variable was also created by combining depressed mood and anhedonia (i.e., scored "1" if either were endorsed). Correlations were 0.09 and -0.04 for NSSI and SA, respectively. Hence, only anhedonia, which was significantly correlated with self-harm was included in discordant twin models.
}

(Table 2; columns 2 and 3). Chi-square tests revealed no significant differences between the prevalence rates of SA and NSSI by EAU status, nor were their differences in self-harm between twins in concordant and discordant pairs who endorsed EAU (columns 4 and 5). However, within the discordant pairs, EAU was associated with significantly higher rates of both SA, $\chi^{2}(1, N=1,396)=6.93, p<0.01$, and NSSI, $\chi^{2}(1, N=1,396)=14.40, p<0.01$, relative to prevalence rates of self-harm for the non-EAU cotwins (columns 3 and 4).

\section{EAU and Risk for Self-Harm}

The mean discordance within twin pairs for EAU was 3.61 years. Within the discordant pairs (Table 3), the twin reporting EAU was at nearly 3-fold increased likelihood of also reporting NSSI $(\mathrm{OR}=2.75,95 \%$ CI: 1.65 to 4.59 , $p<0.01)$ relative to their later onset cotwin. A significant elevation in odds for $\mathrm{SA}$ was also observed $(\mathrm{OR}=2.40,95 \%$ CI: 1.31 to $4.38, p<0.01)$. When adjusting for covariates, EAU resulted in a nearly 8 -fold increased likelihood of NSSI $(\mathrm{OR}=7.62,95 \%$ CI: 2.58 to $22.54, p<0.01)$ and an almost 4-fold increased likelihood of SA (OR $=3.72,95 \%$ CI: 1.19 to $11.64, p<0.05$ ), although the confidence limits around these point estimates were wide. Only anhedonia emerged as a significant covariate for SA. All parameter estimates and test statistics are reported in Table S1.

Finally, the zygosity interaction term, reflecting whether the strength of the association differed across discordant $\mathrm{MZ}$ and DZ pairs was not significant for SA $(p=0.96)$ and was nominally significant for NSSI $(p=0.054)$. Therefore, we examined the association between EAU and NSSI separately for MZ $(n=363)$ and $\mathrm{DZ}(n=335)$ twin pairs (results presented in Table 3 ). The association was significant for the DZ pairs alone $(\mathrm{OR}=4.86,95 \% \mathrm{CI}$ : 2.15 to 10.96, $p<0.01$; see Table S3 for parameter estimates and test statistics).

As the zygosity difference indicated the role of overlapping genetic but not individual-specific environmental influences on the covariance between EAU and NSSI, we fit a bivariate twin model in Mx (Neale et al., 2003) to all available samesex twin data. EAU was moderately heritable $(0.33,95 \% \mathrm{CI}$ : 0.10 to 0.57 ) with the remainder of the variance attributable to shared (0.22, [95\% CI: 0.02 to 0.41$])$ and individual-specific environmental ( 0.45 , [95\% CI: 0.35 to 0.60$])$ factors. Consistent with prior work, $54 \%$ (95\% CI: 0.40 to 0.65$)$ of the variance in NSSI could be attributed to broad sense heritability (additive and dominant genetic influences; see Maciejewski et al., 2014) with the remainder accounted for by individual-specific environmental factors $(0.46$ [95\% CI: 0.35 to 0.60$])$. In line with the findings from the discordant twin modeling, a moderate genetic correlation emerged between EAU and NSSI ( $r_{\mathrm{G}}=0.35,95 \%$ CI: 0.14 to 0.70$)$. The corresponding environmental correlation was modest $\left(r_{\mathrm{E}}=0.24,95 \%\right.$ CI: 0.05 to 0.42$)$. Power to resolve whether genetic or individual-specific environmental influences exclu- 
Table 1. Prevalence (\%) and $95 \%$ Confidence Limits of EAU and Clinical Covariates Among Twins With and Without a History of Self-Harm

\begin{tabular}{|c|c|c|c|c|}
\hline & \multicolumn{2}{|c|}{ NSSI } & \multicolumn{2}{|c|}{ SA } \\
\hline & Present & Absent & Present & Absent \\
\hline Early alcohol use (EAU) & 36.93 (31.52 to 42.34$)$ & 19.55 (18.52 to 20.57 ) & 36.95 (30.31 to 43.59 ) & 19.97 (18.94 to 20.99$)$ \\
\hline Depressed mood & 26.47 (21.53 to 31.41$)$ & 25.43 (24.31 to 26.56$)$ & $19.70(14.23$ to 25.18$)$ & 25.56 ( 24.44 to 0.26 .68 \\
\hline Anhedonia & 16.67 (12.49 to 20.84$)$ & 3.95 (3.45 to 4.45$)$ & 15.76 (10.75 to 20.78$)$ & 3.96 (3.46 to 4.46$)$ \\
\hline Childhood sexual abuse & $19.28(14.86$ to 23.70$)$ & 6.58 (5.94 to 7.22$)$ & 30.54 (24.29 to 37.38$)$ & 6.16 (5.54 to 6.77$)$ \\
\hline Family history of SA & 19.93 (15.46 to 24.41$)$ & 8.95 (8.21 to 9.69) & 18.23 (12.92 to 23.54$)$ & 9.11 (8.37 to 9.85$)$ \\
\hline $\begin{array}{l}\text { Family history of excessive } \\
\text { drinking }\end{array}$ & 34.31 (28.99 to 39.63 ) & 24.74 (23.63 to 25.85$)$ & 38.92 (32.21 to 45.62$)$ & 24.67 (23.56 to 25.77 ) \\
\hline \multicolumn{5}{|l|}{ Risky behavior } \\
\hline 1 & 38.89 (33.43 to 44.35$)$ & 37.00 (35.75 to 38.24$)$ & 35.96 (29.36 to 42.56$)$ & 37.26 (36.01 to 38.50$)$ \\
\hline 2 & 22.22 ( 17.56 to 26.88 ) & 17.04 (16.07 to 18.03$)$ & $25.12(19.16$ to 31.09$)$ & $16.95(15.98$ to 17.91$)$ \\
\hline 3 & 17.32 (13.08 to 21.56$)$ & 7.70 (7.02 to 8.39$)$ & 17.24 (12.05 to 22.44$)$ & 7.75 (7.07 to 8.44$)$ \\
\hline 4 & 4.58 (2.52 to 7.56$)$ & 2.30 (1.92 to 2.69$)$ & 4.93 (1.95 to 7.90$)$ & 2.23 (1.85 to 2.61$)$ \\
\hline
\end{tabular}

SA, suicide attempt; NSSI, nonsuicidal self-injury; EAU, prior to age 15; covariates were only considered present if occurring prior to or within the same year as EAU; Risky behavior, the number of risky behaviors endorsed (i.e., ever use of marijuana, smoking a cigarette a day at least 1 day a week for 3 or more weeks, early voluntary sexual intercourse, and 1+ conduct disorder symptoms).

Table 2. Prevalence (\%) and 95\% Confidence Limits of Self-Harm Among All Twins, Concordant +, Concordant-, and Discordant Early Alcohol Users Versus Discordant Non-EAU Cotwin

\begin{tabular}{|c|c|c|c|c|c|}
\hline & All twins & $\begin{array}{l}\text { Concordant for no EAU } \\
\text { use }(n=4,142)\end{array}$ & $\begin{array}{l}\text { No EAU twin from } \\
\text { disc. pairs }(n=698)\end{array}$ & $\begin{array}{l}\text { EAU twin from disc. } \\
\text { pairs }(n=698)\end{array}$ & $\begin{array}{l}\text { Concordant for } \\
\text { EAU }(n=544)\end{array}$ \\
\hline $\begin{array}{l}\text { Suicide attempt } \\
\qquad(n=6,033)\end{array}$ & 3.36 (2.91 to 3.82 ) & $2.58^{\mathrm{a}}(2.10$ to 3.07$)$ & $3.18^{\mathrm{a}}(1.87$ to 4.49$)$ & $6.17^{b}(4.38$ to 7.96$)$ & $5.90^{b}$ (3.92 to 7.89$)$ \\
\hline $\begin{array}{l}\text { Nonsuicidal } \\
\text { self-injury }(n=6,082)\end{array}$ & 5.03 (4.50 to 5.61$)$ & $3.98^{\mathrm{a}}$ (3.39 to 4.58$)$ & $4.01^{\mathrm{a}}$ (2.56 to 5.47$)$ & $9.03^{\mathrm{b}}(6.90$ to 11.15$)$ & $9.19^{b}$ (6.76 to 11.62$)$ \\
\hline
\end{tabular}

disc, discordant; EAU, early alcohol use.

Similar superscripts $(a, b)$ in rows indicate prevalence rates that could be statistically equated to each other based on chi-square tests.

Table 3. Odds of Lifetime Self-Harm in the Early Alcohol Using Twin Relative to the Later Using Twin

\begin{tabular}{|c|c|c|c|}
\hline Outcome & $\begin{array}{l}\text { Unadjusted } \\
\text { conditional } \\
\text { odds ratio }\end{array}$ & $\begin{array}{l}\text { Adjusted } \\
\text { conditional } \\
\text { odds ratio }\end{array}$ & $\begin{array}{l}\text { Significant } \\
\text { covariates }\end{array}$ \\
\hline $\mathrm{SA}(n=1,388)$ & $\begin{array}{c}2.40^{\star *} \\
(1.31 \text { to } 4.38)\end{array}$ & $\begin{array}{c}3.72^{*} \\
\text { (1.19 to } 11.64)\end{array}$ & Anhedonia* \\
\hline NSSI $(n=1,396)$ & $\begin{array}{c}2.75^{\star *} \\
\text { (1.65 to } 4.59)\end{array}$ & $\begin{array}{c}7.62^{\star \star} \\
\text { (2.58 to } 22.54)\end{array}$ & None \\
\hline NSSI (MZ) $(n=726)$ & $\begin{array}{c}1.62 \\
(0.81 \text { to } 3.23)\end{array}$ & - & - \\
\hline NSSI (DZ) $(n=670)$ & $\begin{array}{c}4.86^{\star \star} \\
\text { (2.15 to } 10.96)\end{array}$ & - & - \\
\hline
\end{tabular}

${ }^{*} p<0.05 ;{ }^{*} p<0.01 ;$ SA, suicide attempt; NSSI, nonsuicidal self-injury; MZ, monozygotic; DZ, dizygotic; Adjusted model covariates include: anhedonia, childhood sexual abuse, risky behavior (4 dummy coded variables representing the number of risky behaviors endorsed: marijuana use, early smoking, early sex, conduct problems).

sively influenced the covariation was limited as both $\mathrm{r}_{\mathrm{G}}$ and $\mathrm{r}_{\mathrm{E}}$ could be individually constrained to zero but not both.

We also conducted a number of sensitivity analyses. To examine whether our results were sensitive to the age threshold for EAU, we reran the discordant twin analyses using age cutoffs of $\leq 13$ and $\leq 15$ years. Due to power limitations, the SA model for $\leq 13$ years could not be estimated, but for $\leq 15$ years, the adjusted OR (3.45) was significant and highly comparable to the existing results. Adjusted ORs were signif- icant for NSSI using both age cutoffs: $\leq 13(\mathrm{OR}=10.91)$ and $\leq 15$ (OR = 3.56), which is also consistent with the current findings. Notably, confidence limits around point estimates were extremely wide when $\leq 13$ years was used for the EAU cutoff, suggesting that our choice of $\leq 14$ years optimizes power while also capturing individuals with early onsets. Additional analyses examined whether results from the discordant twin models were sex or cohort specific. Neither the interaction of EAU with sex nor with cohort was significant indicating that the results generalize to both men and women and across both twin samples.

\section{DISCUSSION}

Self-harm is associated with considerable costs, individually, socially, and economically, and therefore, understanding its etiology is critical to identify potential intervention targets. This study was the first, to our knowledge, to examine mechanisms underlying the association between EAU and self-harm, while controlling for shared environmental and genetic influences. Broadly, prevalence rates of lifetime self-harm in this study were comparable to those reported in meta-analytic work using adult samples (Nock et al., 2008; Swannell et al., 2014), but they were somewhat lower than those reported in adolescent samples (Nock et al., 2013; 
Swannell et al., 2014). This discrepancy between lifetime selfharm (and particularly NSSI) in adolescents relative to adults is perplexing, and potential explanations for this include recent increases in adolescent self-harm and/or retrospective reporting bias by adults, the latter of which is more consistent with existing literature (see Nock et al., 2008). The results of this study also confirm the established overlap between NSSI and SA (Hamza et al., 2012), as approximately $13 \%$ of those endorsing lifetime self-harm (i.e., 59 of 450) reported a history of both SA and NSSI.

In terms of the link between EAU and self-harm, the prevalence of EAU was substantially higher among those with a reported history of SA or NSSI, and, even after controlling for other potential early risk factors (e.g., childhood sexual abuse, conduct disorder, anhedonia, early drug use, family history of suicide, etc.), EAU was significantly associated with increased risk for both NSSI $(\mathrm{OR}=2.71)$ and SA $(\mathrm{OR}=2.56)$. These results confirm previous findings linking EAU to SA (e.g., Bossarte and Swahn, 2011; Swahn et al., 2010). Although, there is less research examining EAU in relation to NSSI, the current findings are consistent with research in high-risk adolescent samples demonstrating that alcohol use predicts NSSI at a 1-year follow-up (Tuisku et al., 2014).

The prevalence of self-harm was also examined in the full sample and across 4 groups, which differed in concordance and exposure to EAU. The prevalence of SA and NSSI was significantly higher in twin pairs concordant for EAU and in the EAU exposed twin in discordant pairs relative to twin pairs concordant for no EAU and unexposed twins in discordant twin pairs. However, there were no significant differences in the latter 2 groups, which would be expected if shared predispositions were strongly influencing the co-occurrence of EAU and self-harm. Importantly, the elevated rates of both SA and NSSI among EAU exposed twins from discordant pairs relative to their unexposed cotwin suggest that the link between these phenotypes is either causal or due to individual-specific factors. In other words, if there were shared familial factors strongly influencing EAU and selfharm, it would be expected that twin pairs - who share genes and the familial environment - would exhibit similar rates of self-harm regardless of exposure to EAU, which was not the case. Importantly, however, these analyses did not differentiate between MZ and DZ discordant pairs to examine whether the association between EAU and self-harm depends on the level of genetic similarity, nor did they did take into account other clinical variables (e.g., conduct disorder) which may be influencing this relationship. Therefore, more rigorous tests of the link between EAU and both SA and NSSI were examined among discordant twin pairs when controlling for zygosity and other clinical covariates.

In these more stringent analyses within discordant twin pairs, the risk for SA among those reporting EAU was elevated nearly 4-fold even after controlling for other potentially causal influences (e.g., childhood sexual abuse, other substance use). This elevated risk for SA among twins who reported drinking prior to age 15 could reflect individualspecific factors that precede EAU, such as other traumatic childhood events or different peer groups that facilitate risky behavior. For example, Trucco and colleagues (2011) found that peer delinquency prospectively predicts peer approval of alcohol and peer use, which in turn predicted initiation of alcohol use among adolescents. Delinquency during adolescence has been linked to a number of maladaptive outcomes, including less positive parenting (Trucco et al., 2011), and furthermore, lack of perceived familial support has been associated with SA (Miller et al., 2015; Wolff et al., 2013). Therefore, these unshared individual-specific influences may be critical in understanding the link between EAU and SA. However, these findings also support the possibility of a causal pathway from EAU to SA. This is consistent with epidemiological research in multiple countries implicating EAU among boys and girls as a risk factor for SA (Kim and Kim, 2010; Swahn and Bossarte, 2007; Swahn et al., 2010, 2012). All of these studies controlled for additional childhood risk factors, but importantly, did not control for shared familial influences. Thus, this study enables the assertion that the link between EAU and SA is potentially independent from genetic or shared environmental factors.

With this said, we did not examine intermediate variables, or individual-specific consequences of EAU, that may explain the causal link between EAU and SA. For example, it is possible that EAU may exert risk for SA via the development of more problematic alcohol use, such as an alcohol use disorder, which is elevated among those reporting early onset drinking (Grant and Dawson, 1997; Sartor et al., 2007). Furthermore, the median age of onset of SA in this study is identical to the median age of onset for both alcohol abuse and dependence in the Australian population (i.e., 20 years; Teesson et al., 2010), which indicates that alcohol use disorder may be an important phenotype to consider in this pathway. It is also possible that EAU contributes to alterations in brain regions that influence response inhibition, which in turn increases risk for later SA. Wetherill and colleagues (2013) found differences in activation of brain regions associated with inhibitory control (e.g., frontal regions) both before and after the onset of heavy drinking among adolescents relative to nondrinking controls. Alterations in frontal brain regions associated with decision-making/inhibition have also been identified among individuals with a history of SA (for a review, see van Heeringen et al., 2011). Therefore, future studies should examine these potential mediators explaining the linking between EAU and SA.

For NSSI, results from the discordant twin analysis demonstrated substantially increased risk for twins reporting EAU $(\mathrm{OR}=7.62)$. However, odds for NSSI were increased only for DZ twins who reported EAU, but not for MZ twins, thereby suggesting that correlated genes may explain the association between EAU and NSSI. These findings indicate that differences in prevalence rates of NSSI between EAU exposed and unexposed cotwins were likely driven by genes unshared by DZ twins. The nonsignificant association in $\mathrm{MZ}$ 
twins pairs is inconsistent with bivariate twin analyses demonstrating a significant environmental correlation between EAU and NSSI $\left(r_{\mathrm{E}}=0.24\right)$, which could reflect limited power in the discordant twin analyses. In line with the discordant results, however, the bivariate twin analyses indicated that both EAU and NSSI were influenced by genetic factors, and furthermore, the significant genetic correlation showed that a portion of these genetic influences are shared $\left(r_{\mathrm{G}}=0.35\right)$. Notably, previous work has found limited evidence for genetic influences on EAU and instead exclusively highlighted the role of unique and shared environmental factors (Richmond-Rakerd et al., 2014). Alternatively, results from this study, which did not include opposite-sex pairs or unpaired twins, are consistent with research demonstrating that both EAU (heritability $=0.36$; Young-Wolff et al., 2012) and NSSI (heritability $=0.59$ for women and 0.37 for men; Maciejewski et al., 2014) are partially influenced by genetic factors. One plausible explanation for these results is that this shared genetic liability may reflect vulnerability to other forms of psychopathology, such as borderline personality disorder, or to dispositional risk factors not assessed in this study, namely disinhibition or negative urgency, a facet of impulsivity that manifests as a tendency to engage in rash behavior when experiencing negative emotions (Whiteside and Lynam, 2003). For example, Squeglia and colleagues (2014) have demonstrated that poor inhibitory control predicts early adolescent transition to alcohol use. Self-reported impulsivity, and more specifically negative urgency, has also been linked to self-harm (Hamza et al., 2015), although these findings do not translate to laboratory-based measures of impulsivity. Therefore, future research should examine whether the association between EAU and self-harm remains when controlling for personality traits and other dispositional factors.

There are several limitations of this study, namely the use of retrospective reporting of both self-harm and age at first drink, the latter of which may be susceptible to reporting bias (Sartor et al., 2011). In addition, due to the lack of assessment of the age of onset of NSSI, the hypothesis that EAU exerts a causal influence on subsequent NSSI could not be fully tested (i.e., participants who engaged in NSSI prior to their first drink could be included in the analyses). Future studies would benefit from more detailed assessment of the time of initiation of these self-harm behaviors relative to initiation of alcohol use. Larger sample sizes could also confirm the differential odds of NSSI for MZ and DZ twins endorsing EAU, which would substantiate the lack of a causal pathway between these phenotypes observed in this study. Additionally, due to lack of power, it was not possible to examine potential differences in the link between EAU and subgroups of individuals engaging in SA. A previous study has established that cannabis use is associated with increased odds for SA, but only for unplanned rather than planned attempts (Delforterie et al., 2015). Therefore, it may be the case that other substance use, such as EAU, may be related to certain types of SA. It is also possible that NSSI and SA were somewhat confounded in this study, in that only about half of individuals endorsing SA reported intent to die. Therefore, future studies should examine the association between EAU and SA with intent relative to SA without intent. Finally, this study was a homogenous Caucasian sample from Australia, so these findings may not generalize to other samples, particularly given research demonstrating differences in the association between NSSI and substance use across cultures (Giletta et al., 2012) and in the heritability of age at first drink for African Americans and European Americans (Sartor et al., 2013).

In conclusion, the results of this study confirm previous research demonstrating an association between EAU and self-harm. Specifically, the findings suggest that shared genes influence the link between EAU and NSSI, and that there may be a putatively causal link between EAU and SA. This highlights the need for further studies aimed at identifying genetic factors (e.g., predisposition to negative urgency) that contribute to the overlap between EAU and NSSI and through which EAU may causally influence SA (e.g., brain development). The results also suggest that interventions aimed at delaying initiation of alcohol use may be influential in minimizing risk for SA.

\section{DECLARATIONS OF INTEREST}

This research was funded by National Institute on Alcohol Abuse and Alcoholism (NIAAA) grants: AA023693 (LRF); AA21235 (AA); AA11998, AA07728, and AA13221 (ACH); AA017921, AA023549 (CES); National Institute on Drug Abuse (NIDA) grants: DA18267 (MTL) facilitated through access to the Australian Twin Registry, a national resource supported by an Enabling Grant (ID 628911) from the National Health \& Medical Research Council. NGM acknowledges support from the Australian NHMRC Centre for Research Excellence on Suicide Prevention (CRESP, PI Dr. Helen Christensen). CES acknowledges support from the Robert E. Leet and Clara Guthrie Patterson Trust. AA has received peer-reviewed grant funding, travel reimbursements and an honorarium from ABMRF/Foundation for Alcohol Research, which receives some of its funds from brewers. There are no conflicts of interest to report.

\section{REFERENCES}

Agrawal A, Sartor CE, Lynskey MT, Grant JD, Pergadia ML, Grucza R, Bucholz KK, Nelson EC, Madden PA, Martin NG, Heath AC (2009) Evidence for an interaction between age at first drink and genetic influences on DSM-IV alcohol dependence symptoms. Alcohol Clin Exp Res 33:2047-2056.

Bagge CL, Sher KJ (2008) Adolescent alcohol involvement and suicide attempts: toward the development of a conceptual framework. Clin Psychol Rev 28:1283-1296.

Bebbington PE, Minot S, Cooper C, Dennis M, Meltzer H, Jenkins R, Brugha T (2010) Suicidal ideation, self-harm and attempted suicide: results 
from the British psychiatric morbidity survey 2000. Eur Psychiatry 25:427-431.

Bossarte RM, Swahn MH (2011) The associations between early alcohol use and suicide attempts among adolescents with a history of major depression. Addict Behav 36:532-535.

Conner KR, Bagge CL, Goldston DB, Ilgen MA (2014) Alcohol and suicidal behavior: what is known and what can be done. Am J Prev Med 47(3 Suppl 2):S204-S208.

Delforterie MJ, Lynskey MT, Huizink AC, Creemers HE, Grant JD, Few LR, Glowinski AL, Statham DJ, Trull TJ, Bucholz KK, Madden PA, Martin NG, Heath AC, Agrawal A (2015) The relationship between cannabis involvement and suicidal thoughts and behaviors. Drug Alcohol Depend 150:98-104.

Dir AL, Karyadi K, Cyders MA (2013) The uniqueness of negative urgency as a common risk factor for self-harm behaviors, alcohol consumption, and eating problems. Addict Behav 38:2158-2162.

GBD 2010 Country Collaboration (2013) GBD 2010 country results. Lancet 381:965-970.

Giletta M, Scholte RH, Engels RC, Ciairano S, Prinstein MJ (2012) Adolescent non-suicidal self-injury: a cross-national study of community samples from Italy, the Netherlands and the United States. Psychiatry Res 197:6672 .

Glowinski AL, Bucholz KK, Nelson EC, Fu Q, Madden PA, Reich W, Heath AC (2001) Suicide attempts in an adolescent female twin sample. J Am Acad Child Adolesc Psychiatry 40:1300-1307.

Grant BF, Dawson DA (1997) Age at onset of alcohol use and its association with DSM-IV alcohol abuse and dependence: results from the National Longitudinal Alcohol Epidemiologic Survey. J Subst Abuse 9:103-110.

Grant JD, Scherrer JF, Lynskey MT, Agrawal A, Duncan AE, Haber JR, Heath AC, Bucholz KK (2012) Associations of alcohol, nicotine, cannabis, and drug use/dependence with educational attainment: evidence from cotwin-control analyses. Alcohol Clin Exp Res 36:1412-1420.

Guerri C, Pascual M (2010) Mechanisms involved in the neurotoxic, cognitive, and neurobehavioral effects of alcohol consumption during adolescence. Alcohol 44:15-26.

Hamza CA, Stewart SL, Willoughby T (2012) Examining the link between nonsuicidal self-injury and suicidal behavior: a review of the literature and an integrated model. Clin Psychol Rev 32:482-495.

Hamza CA, Willoughby T, Heffer T (2015) Impulsivity and nonsuicidal selfinjury: a review and meta-analysis. Clin Psychol Rev 38:13-24.

van Heeringen C, Bijttebier S, Godfrin K (2011) Suicidal brains: a review of functional and structural brain studies in association with suicidal behaviour. Neurosci Biobehav Rev 35:688-698.

Hingson R, White A (2014) New research findings since the 2007 surgeon general's call to action to prevent and reduce underage drinking: a review. J Stud Alcohol Drugs 75:158-169.

Kaplan MS, Huguet N, McFarland BH, Caetano R, Conner KR, Giesbrecht N, Nolte KB (2014) Use of alcohol before suicide in the United States. Ann Epidemiol 24:588-592.

Kessler RC, Borges G, Walters EE (1999) Prevalence of and risk factors for lifetime suicide attempts in the National Comorbidity Survey. Arch Gen Psychiatry 56:617-626.

Kim DS, Kim HS (2010) Early initiation of alcohol drinking, cigarette smoking, and sexual intercourse linked to suicidal ideation and attempts: findings from the 2006 Korean Youth Risk Behavior Survey. Yonsei Med J $51: 18-26$

Klonsky ED (2011) Non-suicidal self-injury in United States adults: prevalence, sociodemographics, topography and functions. Psychol Med 41:1981-1986.

Larkin C, Di BZ, Arensman E (2014) Risk factors for repetition of self-harm: a systematic review of prospective hospital-based studies. PLoS One 9: e84282.

Lynskey MT, Agrawal A, Henders A, Nelson EC, Madden PA, Martin NG (2012) An Australian twin study of cannabis and other illicit drug use and misuse, and other psychopathology. Twin Res Hum Genet 15:631-641.
Lynskey MT, Heath AC, Bucholz KK, Slutske WS, Madden PA, Nelson EC, Statham DJ, Martin NG (2003) Escalation of drug use in early-onset cannabis users vs co-twin controls. JAMA 289:427-433.

Maciejewski DF, Creemers HE, Lynskey MT, Madden PA, Heath AC, Statham DJ, Martin NG, Verweij KJ (2014) Overlapping genetic and environmental influences on nonsuicidal self-injury and suicidal ideation: different outcomes, same etiology? JAMA Psychiatry 71:699-705.

Miller AB, Esposito-Smythers C, Leichtweis RN (2015) Role of social support in adolescent suicidal ideation and suicide attempts. J Adolesc Health $56: 286-292$

Moller CI, Tait RJ, Byrne DG (2013) Self-harm, substance use and psychological distress in the Australian general population. Addiction 108:211220.

Neale MC, Boker SM, Xie G, Maes HH (2003) Mx: Statistical Modeling. 6th ed. Department of Psychiatry, Richmond.

Nock MK, Borges G, Bromet EJ, Alonso J, Angermeyer M, Beautrais A, Bruffaerts R, Chiu WT, de Girolamo G, Gluzman S, deGraaf R, Gureje O, Haro JM, Huang Y, Karam E, Kessler RC, Lepine JP, Levinson D, Medina-Mora ME, Ono Y, Posada-Villa J, Williams D (2008) Cross-national prevalence and risk factors for suicidal ideation, plans and attempts. Br J Psychiatry 192:98-105.

Nock MK, Favazza A (2009) Non-suicidal self-injury: definition and classification, in Understanding Non-Suicidal Self-Injury: Origins, Assessment, and Treatment (Nock MK ed), pp 9-18. American Psychological Association, Washington, DC.

Nock MK, Green JG, Hwang I, McLaughlin KA, Sampson NA, Zaslavsky AM, Kessler RC (2013) Prevalence, correlates, and treatment of lifetime suicidal behavior among adolescents: results from the National Comorbidity Survey Replication Adolescent Supplement. JAMA Psychiatry 70:300310.

Nock MK, Joiner TE Jr, Gordon KH, Lloyd-Richardson E, Prinstein MJ (2006) Non-suicidal self-injury among adolescents: diagnostic correlates and relation to suicide attempts. Psychiatry Res 144:65-72.

Patton GC, Coffey C, Sawyer SM, Viner RM, Haller DM, Bose K, Vos T, Ferguson J, Mathers CD (2009) Global patterns of mortality in young people: a systematic analysis of population health data. Lancet 374:881892.

Prescott CA, Kendler KS (1999) Age at first drink and risk for alcoholism: a noncausal association. Alcohol Clin Exp Res 23:101-107.

Richmond-Rakerd LS, Slutske WS, Heath AC, Martin NG (2014) Genetic and environmental influences on the ages of drinking and gambling initiation: evidence for distinct aetiologies and sex differences. Addiction 109:323-331.

Sartor CE, Agrawal A, Lynskey MT, Bucholz KK, Madden PA, Heath AC (2009a) Common genetic influences on the timing of first use for alcohol, cigarettes, and cannabis in young African-American women. Drug Alcohol Depend 102:49-55.

Sartor CE, Bucholz KK, Nelson EC, Madden PA, Lynskey MT, Heath AC (2011) Reporting bias in the association between age at first alcohol use and heavy episodic drinking. Alcohol Clin Exp Res 35:1418-1425.

Sartor CE, Lynskey MT, Bucholz KK, Madden PA, Martin NG, Heath AC (2009b) Timing of first alcohol use and alcohol dependence: evidence of common genetic influences. Addiction 104:1512-1518.

Sartor CE, Lynskey MT, Heath AC, Jacob T, True W (2007) The role of childhood risk factors in initiation of alcohol use and progression to alcohol dependence. Addiction 102:216-225.

Sartor CE, Nelson EC, Lynskey MT, Madden PA, Heath AC, Bucholz KK (2013) Are there differences between young African-American and European-American women in the relative influences of genetics versus environment on age at first drink and problem alcohol use? Alcohol Clin Exp Res 37:1939-1946.

Squeglia LM, Jacobus J, Nguyen-Louie TT, Tapert SF (2014) Inhibition during early adolescence predicts alcohol and marijuana use by late adolescence. Neuropsychology 28:782-790.

Stewart SE, Manion IG, Davidson S, Cloutier P (2001) Suicidal children and adolescents with first emergency room presentations: predictors of sixmonth outcome. J Am Acad Child Adolesc Psychiatry 40:580-587. 
Stueve A, O’Donnell LN (2005) Early alcohol initiation and subsequent sexual and alcohol risk behaviors among urban youths. Am J Public Health 95:887-893.

Swahn MH, Bossarte RM (2007) Gender, early alcohol use, and suicide ideation and attempts: findings from the 2005 youth risk behavior survey. J Adolesc Health 41:175-181.

Swahn MH, Bossarte RM, Ashby JS, Meyers J (2010) Pre-teen alcohol use initiation and suicide attempts among middle and high school students: findings from the 2006 Georgia Student Health Survey. Addict Behav 35:452-458.

Swahn MH, Bossarte RM, Choquet M, Hassler C, Falissard B, Chau N (2012) Early substance use initiation and suicide ideation and attempts among students in France and the United States. Int J Public Health 57:95-105.

Swahn MH, Bossarte RM, Palmier JB, Yao H (2013) Co-occurring physical fighting and suicide attempts among U.S. high school students: examining patterns of early alcohol use initiation and current binge drinking. West $\mathbf{J}$ Emerg Med 14:341-346.

Swannell SV, Martin GE, Page A, Hasking P, St John NJ (2014) Prevalence of nonsuicidal self-injury in nonclinical samples: systematic review, metaanalysis and meta-regression. Suicide Life Threat Behav 44:273-303.

Teesson M, Hall W, Slade T, Mills K, Grove R, Mewton L, Baillie A, Haber P (2010) Prevalence and correlates of DSM-IV alcohol abuse and dependence in Australia: findings of the 2007 National Survey of Mental Health and Wellbeing. Addiction 105:2085-2094.

Trucco EM, Colder CR, Wieczorek WF (2011) Vulnerability to peer influence: a moderated mediation study of early adolescent alcohol use initiation. Addict Behav 36:729-736.

Tuisku V, Kiviruusu O, Pelkonen M, Karlsson L, Strandholm T, Marttunen M (2014) Depressed adolescents as young adults - predictors of suicide attempt and non-suicidal self-injury during an 8-year follow-up. J Affect Disord 152-154:313-319.
Wetherill RR, Squeglia LM, Yang TT, Tapert SF (2013) A longitudinal examination of adolescent response inhibition: neural differences before and after the initiation of heavy drinking. Psychopharmacology 230: 663-671.

Whiteside SP, Lynam DR (2003) Understanding the role of impulsivity and externalizing psychopathology in alcohol abuse: application of the UPPS impulsive behavior scale. Exp Clin Psychopharmacol 11:210-217.

Wolff J, Frazier EA, Esposito-Smythers C, Burke T, Sloan E, Spirito A (2013) Cognitive and social factors associated with NSSI and suicide attempts in psychiatrically hospitalized adolescents. J Abnorm Child Psychol 41:1005-1013.

Young-Wolff KC, Kendler KS, Prescott CA (2012) Shared genetic contributions to early-onset drinking and drinking to cope motives. Addict Behav 37:1176-1180.

\section{SUPPORTING INFORMATION}

Additional Supporting Information may be found in the online version of this article:

Table S1. Results from unadjusted and adjusted conditional logistic regression analyses examining EAU and selfharm in the full sample of same-sex twins and among discordant twin pairs.

Table S2. Tetrachoric correlations and chi-square test results between self-harm variables and clinical covariates.

Table S3. Results of conditional logistic regression analyses examining EAU and NSSI in MZ and DZ twin pairs. 